\title{
Undetectable Tumor Cell-Free DNA in a Patient With Metastatic Breast Cancer With Complete Response and Long-Term Remission
}

Natasha Hunter, $\mathrm{MD}^{1}$; Sarah Croessmann, $\mathrm{PhD}^{2}$; Karen Cravero, $\mathrm{PhD}^{3}$; Daniel Shinn, BS${ }^{3}$; Paula J. Hurley, PhD2,3; and Ben Ho Park, MD, PhD²,3

\begin{abstract}
The ability to serially monitor tumor-derived cell-free DNA (cfDNA) brings with it the potential to measure response to anticancer therapies and detect minimal residual disease (MRD). This report describes a patient with HER2-positive metastatic breast cancer with an exceptional response to trastuzumab and nab-paclitaxel who remains in complete remission several years after cessation of therapy. Next-generation sequencing of the patient's primary tumor tissue showed several mutations, including an oncogenic hotspot PIK3CA mutation. A sample of cfDNA was collected 6 years after her last therapy and then analyzed for mutant PIK3CA using digital PCR. No detectable mutations associated with the primary tumor were found despite assaying $>10,000$ genome equivalents, suggesting that the patient had achieved a molecular remission. Results of this case study suggest that serial monitoring of MRD using liquid biopsies could provide a useful method for individualizing treatment plans for patients with metastatic disease with extreme responses to therapy. However, large-scale clinical studies are needed to validate and implement these techniques for patient care.
\end{abstract}

J Natl Compr Canc Netw 2020;18(4):375-379 doi: $10.6004 /$ jnccn.2019.7381

${ }^{1}$ Fred Hutchinson/University of Washington Cancer Consortium, Seattle, Washington; ${ }^{2}$ Vanderbilt-Ingram Cancer Center, Vanderbilt University Medical Center, Nashville, Tennessee; and ${ }^{3}$ The Sidney Kimmel Comprehensive Cancer Center, Johns Hopkins School of Medicine, Baltimore, Maryland.
Precision oncology focuses on matching targeted therapies to genetic alterations in a patient's tumor, usually in a metastatic setting. When responses to such treatments are seen, the long-term benefit remains uncommon. However, a minority of patients are "exceptional responders," and although no standardized definition of this phenomenon has been established, sustained remissions or lack of progression spanning years to decades has generally characterized this cohort. Presentations vary widely, including stable disease for protracted periods and a few exceptional responders who demonstrate no evidence of metastatic disease even after cessation of antineoplastic therapies. Whether these patients are cured or harbor minimal residual disease (MRD) that may ultimately relapse is unknown.

The use of plasma tumor cell-free DNA (cfDNA) as "liquid biopsies" has been championed for identifying mutations amenable to targeted therapies and for the assessment of MRD. ${ }^{1-4}$ With the advent of droplet digital PCR (ddPCR) and ultra-deep next-generation sequencing (NGS), clinicians are able to track tumor burden, evaluate response to therapies, and detect MRD using cancer DNA alterations present in cfDNA as a biomarker. Indeed, many studies have supported the feasibility of cfDNA for serial monitoring. ${ }^{5}$ For example, we and others have demonstrated that ddPCR can detect MRD using cfDNA in patients with early-stage breast cancer after curative-intent therapies. ${ }^{1,3}$ In addition, we are testing the clinical utility of cfDNA in patients with earlystage, HER2-positive and triple-negative breast cancer in a large-scale, multi-institutional prospective study (ClinicalTrials.gov identifier: NCT02743910).

In this study, we assessed cfDNA from a patient with estrogen receptor (ER)/progesterone receptor (PR)-negative, HER2-positive metastatic breast cancer who received

See JNCCN.org for supplemental online content. 
trastuzumab and nab-paclitaxel in 2008 and experienced a complete remission (CR). Remarkably, she continues to have no evidence of disease several years beyond the cessation of any cancer-directed therapies. NGS of her primary tumor tissue showed several mutations, including a PIK3CA mutation, which was used to assess MRD via cfDNA. We also tested several other tissues to rule out germline variants and clonal hematopoiesis $(\mathrm{CH})$. These findings suggest that long-term blood-based monitoring of cfDNA may distinguish patients with MRD from those without molecular evidence of disease, which could potentially help clinicians evaluate the risk versus benefit of ongoing maintenance therapy for patients with metastatic disease who experience extreme responses to treatment.

\section{Case Report}

A premenopausal woman aged 39 years presented in 2008 with a left-sided breast mass that was further evaluated through physical examination, imaging, and diagnostic biopsy. She was diagnosed with an ER/PR-negative highgrade invasive ductal carcinoma, which was positive for HER2 expression (3+ by immunostaining). Further studies with breast MRI and CT revealed enlarged axillary lymph nodes and numerous bilateral pulmonary nodules suspicious for metastatic disease. Fine-needle aspiration biopsy of a lung lesion confirmed metastatic spread of her primary breast cancer (Figure 1). The patient received 16 weeks of systemic therapy with nab-paclitaxel and trastuzumab, followed by maintenance trastuzumab. In April 2009, CT scans revealed CR of all breast and lung lesions, with the exception of a single lung nodule. This solitary residual nodule was favored to be benign after multiple CT scans demonstrated stability.

In June 2009, the patient remained in remission and elected to undergo mastectomy of the previously affected breast and contralateral prophylactic mastectomy. Pathologic examination of the surgical specimens detected no residual cancer and the patient was advised to continue trastuzumab for at least 1 year total but possibly indefinitely. In October 2011, the patient chose to discontinue trastuzumab secondary to concerns about cardiac toxicity. The patient was advised to continue physical examinations and periodic CT scans for surveillance.

In 2016, germline sequencing was performed using a commercial vendor. Testing revealed no germline variants in 21 common cancer-associated genes, including TP53 and BRCA1/2. In 2017, an archival primary tumor from her diagnostic biopsy was subjected to NGS and

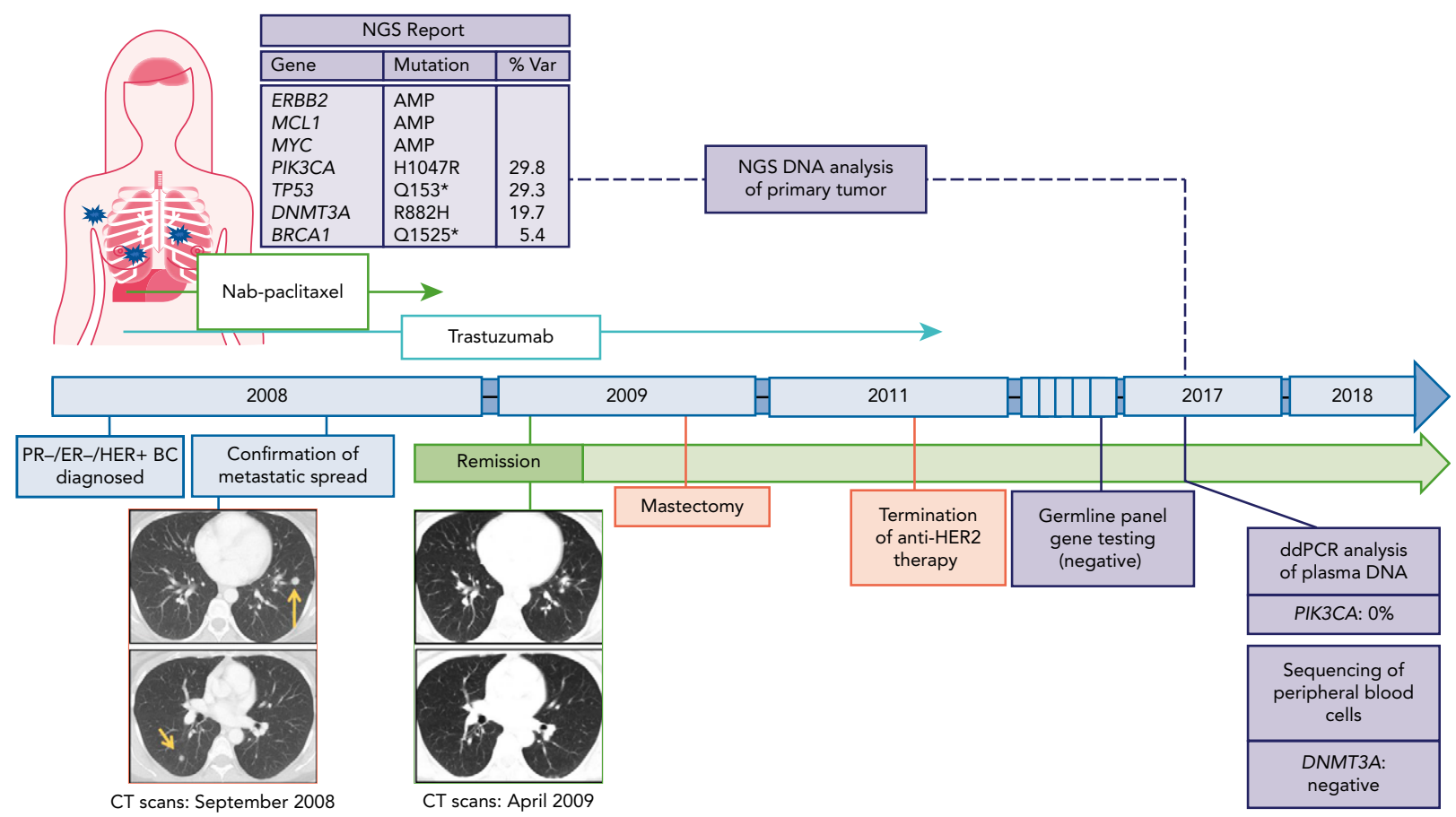

Figure 1. Clinical course of patient with ER- and PR-negative/HER-positive metastatic breast cancer with exceptional response. CT scans in September 2008 showed metastatic spread in lungs (yellow arrows). After treatment with nab-paclitaxel and trastuzumab, CT scans confirmed complete remission in April 2009. The patient underwent germline testing in 2016 and DNA analysis of archival tissue and recently collected samples (plasma, fingernail, buccal swab) in 2017 (purple shaded boxes).

Abbreviations: AMP, amplification; BC, breast cancer; ER, estrogen receptor; ddPCR, droplet digital PCR; NGS, next-generation sequencing; PR, progesterone receptor; var, variant. 
assessed for DNA variants (single nucleotide variants, copy number variants, indels, and fusions) across 144 cancer-associated genes. Unfortunately, archival metastatic biopsy tissue was unavailable for NGS analysis because of the paucity of tissue. However, the pathology report from the fine-needle aspiration biopsy was consistent with metastatic breast cancer from the original primary tumor (see supplemental eAppendix 1, available with this article at JNCCN.org). In addition to HER2 amplification confirming the earlier HER2 immunohistochemistry staining, NGS revealed alterations in 6 other genes (Figure 1), including 2 additional gene amplifications and 4 point mutations. The 4 mutations had relatively high allelic frequencies and included a PIK3CA hotspot mutation (H1047R), a loss-of-function mutation in TP53 (Q153*), a hotspot DNMT3A mutation $(\mathrm{R} 882 \mathrm{H})$, and a subclonal BRCA1 loss-of-function mutation (Q1525*).

The allelic frequency of the TP53 mutation was $29.3 \%$, which did not rule out a germline mutation. However, earlier germline testing was negative for TP53 variants, effectively identifying this mutation as a somatic pathogenic variant. Although PIK3CA and DNMT3A can be mutated in the germline, this type of mutation is extremely rare and generally associated with notable and conspicuous phenotypes. However, to definitively show the somatic nature of these variants, additional samples, including buccal swabs and fingernail clippings, were collected for germline testing and analyzed by Sanger sequencing as described elsewhere ${ }^{6}$ (Figure 2). Results showed only wild-type sequences, strongly suggesting that these hotspot variants were indeed somatic.

Although the DNMT3A mutation is considered a hotspot mutation and is a known driver in hematologic malignancies, it is exceedingly rare in solid tumors and could have represented contamination of blood cells with $\mathrm{CH}^{7}$ Indeed, DNMT3A mutations are one of the most common alterations found in $\mathrm{CH}$, and the $\mathrm{R} 882 \mathrm{H}$ variant in particular has often been identified in $\mathrm{CH}$ cells. $\mathrm{CH}$ is more common in patients aged $>70$ years or in those who have received prior chemotherapy. However, neither of these factors was applicable, given our patient's young age and because the primary sample used for NGS was obtained before any systemic therapies. Nonetheless, sequencing of peripheral lymphocytes was performed and did not reveal the DNMT3A variant, strongly supporting that this mutation did not arise from $\mathrm{CH}$ in the tissue sample but from the breast cancer cells themselves. ${ }^{8}$ Notably, it has been suggested that $\mathrm{CH}$ is associated with worse clinical outcomes in hematologic and solid tumor malignancies, ${ }^{9}$ and given that this patient's extreme favorable response to therapy was inconsistent with $\mathrm{CH}$, this result further supports that the DNMT3A variant was in the breast cancer.

Finally, we assessed the patient's cfDNA using ddPCR for the H1047R PIK3CA mutation. Blood was collected in Streck cfDNA blood collection tubes; it has been shown that using these tubes prevents lysis of white cells, which can lead to artificially high amounts of normal genomic DNA in cfDNA samples. ${ }^{10}$ We chose this mutation to track because it is a common driver mutation in breast cancer, providing rationale that it is a useful surrogate biomarker because it is likely that all derived cancer cells would harbor this mutation. Furthermore, because there was no baseline cfDNA sample from 2008 when the patient first presented, by selecting a predominant or founding mutation detected in the primary biopsy we could confidently correlate cfDNA with tumor burden. Moreover, we have published extensively on the use of ddPCR for detecting this hotspot mutation in patients with both metastatic and early-stage breast cancer, with high sensitivity and specificity. ${ }^{1,4}$ However, lack of a baseline sample is a limitation of the current study. In past studies, we demonstrated accurate detection of PIK3CA mutations of as low as $0.01 \%$ fractional abundance within a cfDNA sample with $93.3 \%$ sensitivity and $100 \%$ specificity. Using this method, we assayed $>10,000$ genome equivalents in this patient's cfDNA and detected no mutations, suggesting that the patient had achieved a molecular remission. As of July 2019, the patient remained in CR with no evidence of disease despite the lack of active cancer treatment.

\section{Discussion}

Since the advent of HER2-targeted therapies, a small population of women with HER2-positive cancers has demonstrated dramatic and durable responses to trastuzumab in the metastatic setting. In fact, one of the first patients with metastatic disease treated on trial using trastuzumab currently remains alive and disease-free. ${ }^{11}$ Unfortunately, to date no reliable biomarkers exist to predict which women will achieve this favorable outcome. In addition, we lack tools to risk-stratify patients with metastatic disease receiving anti-HER2-directed therapies to determine whether they may benefit from ongoing treatment or may safely discontinue their therapies. Thus, current standard of care dictates indefinite and continuous HER2-targeted therapy. This case study highlights that a subset of patients may achieve durable CR from a defined course of anti-HER2-directed therapy and can avoid the risk of cardiac and other associated toxicities posed by long-term treatments. The ability to monitor disease accurately and in real time could provide a method for individualizing therapy for patients with metastatic disease.

The presence of tumor-specific mutations in baseline pretreatment plasma and continued monitoring during treatment could in theory be used to guide therapeutic decisions for patients with both early-stage and metastatic cancers. As shown here, the absence of this patient's PIK3CA mutation in cfDNA was correlated 


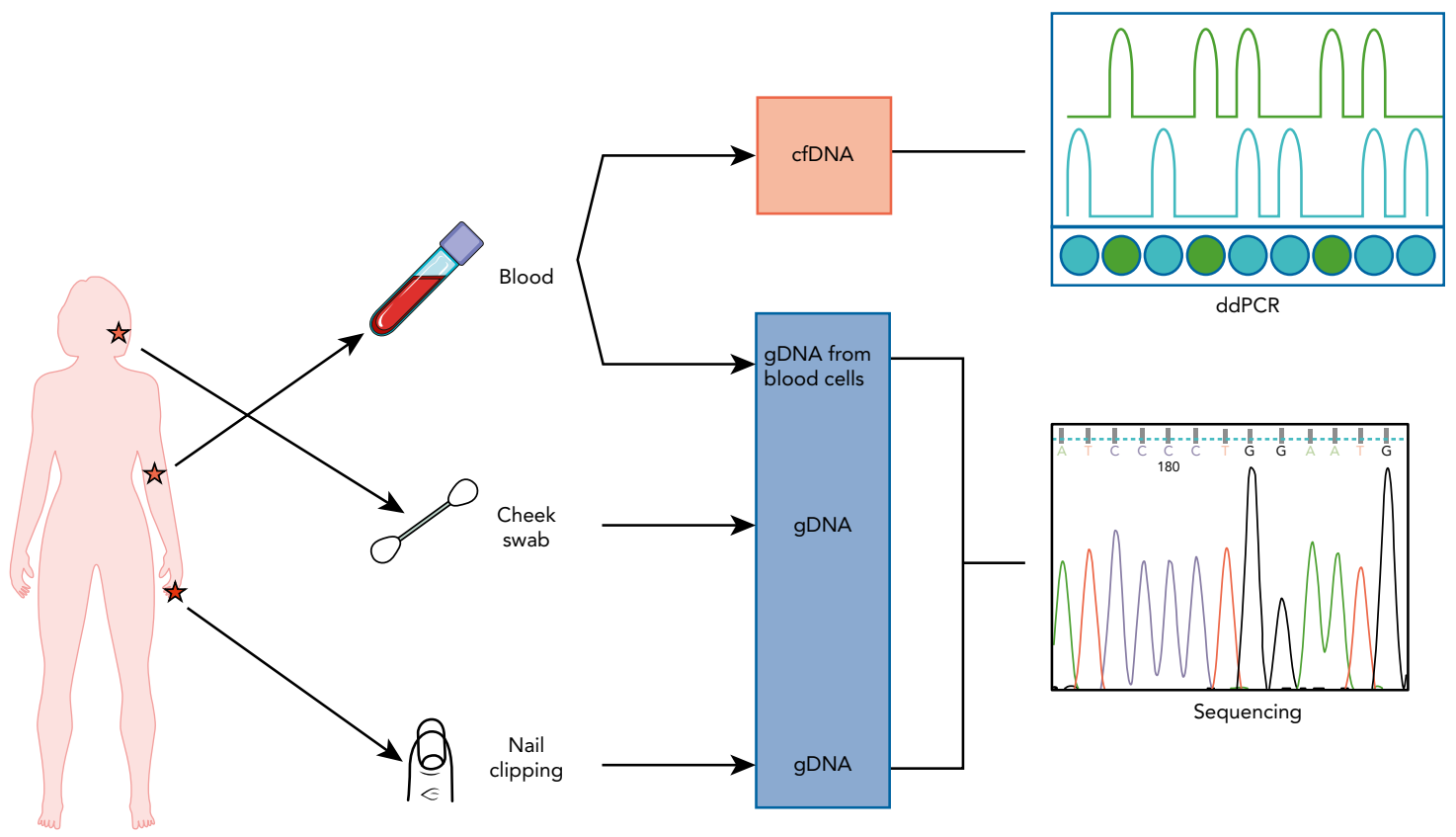

Figure 2. Methodology for DNA analysis of collected samples. Schematic representation of sample processing. Blood samples were analyzed using ddPCR on cfDNA and sequencing using gDNA from peripheral blood cells, buccal swabs, and fingernail clippings.

Abbreviations: cfDNA, cell-free DNA; ddPCR, droplet digital PCR; gDNA, genomic DNA.

with the observed complete response and disease-free survival. Although ddPCR detection of mutations is both highly sensitive and specific, absence of the PIK3CA mutation within a single case study cannot confirm the absence of disease and/or cure. Large-scale, multiinstitutional, prospective studies are ongoing to validate these findings.

\section{Conclusions}

This report presented a patient with metastatic HER2positive breast cancer who had an exceptional response after a fixed duration of systemic therapies. We identified a trackable PIK3CA mutation from her tumor and tested her cfDNA for this variant using ddPCR; this assay proved negative, correlating with the absence of disease despite almost 8 years without systemic therapies. However, understanding and correlating the absence of these mutations with long-term disease-free survival will require large-scale validation, which is a testable hypothesis currently being addressed in clinical trials.

\section{Acknowledgments}

We thank and acknowledge the support of the Nashville Wine Auction, the Parker Foundation, and Susan G. Komen.

Submitted August 2, 2019; accepted for publication November 25, 2019.

Author contributions: Study concept and design: All authors. Development of methodology: Hunter, Croessmann, Cravero, Shinn. Data acquisition: Hunter, Croessmann, Cravero, Shinn, Hurley. Data analysis and interpretation: All authors. Manuscript writing, review, and/or revision: All authors.

Disclosures: Dr. Park has disclosed that he is a scientific advisor for and has ownership interest in Loxo Oncology, and has received grant/research support from AbbVie, Pfizer, and Foundation Medicine, Inc. The remaining authors have not received any financial consideration from any person or organization to support the preparation, analysis, results, or discussion of this article.

Funding: Research reported in this publication was supported by the Breast Cancer Research Foundation (B.H.P.), the American Cancer Society (131356-RSG-17-160-01-CSM; P.J.H.), the Marcie \& Ellen Foundation (N.H. and B.H.P.), and the $\mathrm{NCl}$ of the $\mathrm{NIH}$ under award numbers R01CA214494 (B.H.P.), R01CA194024 (S.C. and B.H.P.), and RO1CA211695 (P.J.H.).

Disclaimer: The content is solely the responsibility of the authors and does not necessarily represent the official views of the NIH.

Correspondence: Ben Ho Park, MD, PhD, Vanderbilt-Ingram Cancer Center, Vanderbilt University Medical Center, 2220 Pierce Avenue, PRB 777, Nashville, TN 37232. Email: ben.h.park@vumc.org

\section{References}

1. Beaver JA, Jelovac D, Balukrishna $S$, et al. Detection of cancer DNA in plasma of patients with early-stage breast cancer. Clin Cancer Res 2014;20:2643-2650.

2. Chu D, Paoletti C, Gersch C, et al. ESR1 mutations in circulating plasma tumor DNA from metastatic breast cancer patients. Clin Cancer Res 2016;22:993-999.
3. Garcia-Murillas I, Schiavon G, Weigelt B, et al. Mutation tracking in circulating tumor DNA predicts relapse in early breast cancer. Sci Transl Med 2015;7:302ra133.

4. Higgins MJ, Jelovac D, Barnathan E, et al. Detection of tumor PIK3CA status in metastatic breast cancer using peripheral blood. Clin Cancer Res 2012;18:3462-3469. 
5. Donaldson J, Park BH. Circulating tumor DNA: measurement and clinical utility. Annu Rev Med 2018;69:223-234.

6. Lee J, Axilbund J, Dalton WB, et al. A polycythemia vera JAK2 mutation masquerading as a duodenal cancer mutation. J Natl Compr Canc Netw 2016;14:1495-1498.

7. Buscarlet M, Provost S, Zada YF, et al. DNMT3A and TET2 dominate clonal hematopoiesis and demonstrate benign phenotypes and different genetic predispositions. Blood 2017;130:753-762.
8. Hu Y, Ulrich BC, Supplee J, et al. False-positive plasma genotyping due to clonal hematopoiesis. Clin Cancer Res 2018;24:4437-4443.

9. Steensma DP. Clinical implications of clonal hematopoiesis. Mayo Clin Proc 2018;93:1122-1130

10. Toro PV, Erlanger B, Beaver JA, et al. Comparison of cell stabilizing blood collection tubes for circulating plasma tumor DNA. Clin Biochem 2015;48:993-998.

11. Mukherjee S. The Emperor of All Maladies: A Biography of Cancer. New York, NY: Scribner; 2010.

\section{See JNCCN.org for supplemental online content.}
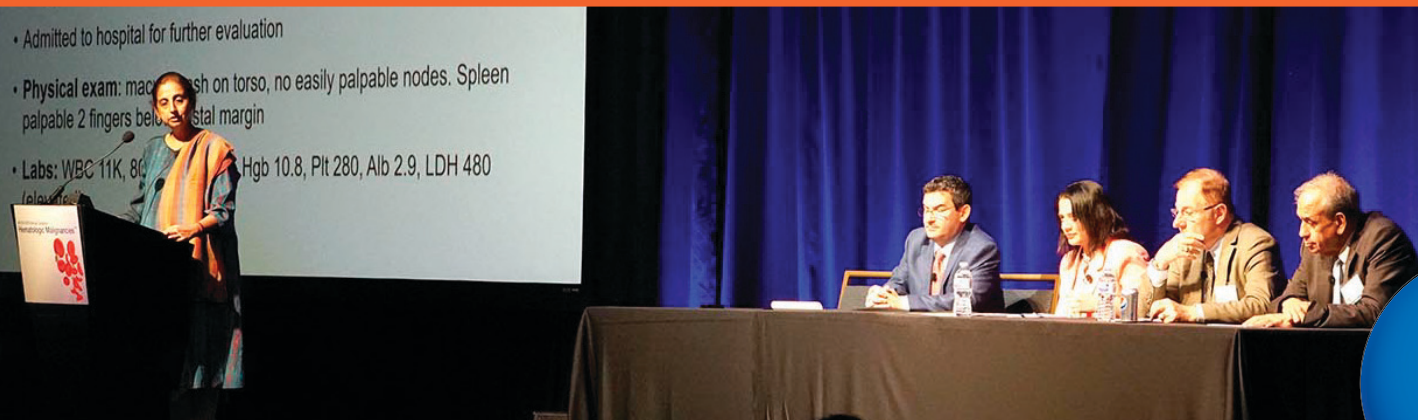

\section{October 9 - 10, 2020 | New York, NY NCCN.org/hem}


Supplemental online content for:

\section{Undetectable Tumor Cell-Free DNA in a Patient With Metastatic Breast Cancer With Complete Response and Long-Term Remission}

Natasha Hunter, MD; Sarah Croessmann, PhD; Karen Cravero, PhD; Daniel Shinn, BS;

Paula J. Hurley, PhD; and Ben Ho Park, MD, PhD

J Natl Compr Canc Netw 2020;18(4):375-379

eAppendix 1: Original Diagnostic Biopsies 


\section{eAppendix 1}

\section{Original Diagnostic Biopsies}

1. Left breast (biopsies, September 8, 2008):

Poorly differentiated infiltrating ductal carcinoma.

Microcalcification identified. See note.

Note: The tumor is ER-negative and PR-negative. Ki67 index: $>30 \%$, high. This case was presented at the daily quality assurance conference.

2. Fine-needle aspiration: needle biopsy, lung, right upper lobe (October 1, 2008):

Final diagnosis: adenocarcinoma, consistent with metastasis from patient's known breast primary. See note.

Note: The accompanying immunostains show the tumor cells to be positive for C-erb-2 (3+) and negative for TTF-1, ER, $\mathrm{PR}$, and p53. This immunoprofile supports the above diagnosis.

Abbreviations: ER, estrogen receptor; PR, progesterone receptor: TTF-1, thyroid transcription factor-1. 